\title{
Mitteilungen der Schweizerischen Gesellschaft für Pathologie
}

Pathologe $2015 \cdot 36: 427$

DOI 10.1007/s00292-015-0044-9

Online publiziert: 14. Juli 2015

○) Springer-Verlag Berlin Heidelberg 2015

\section{SGPath SSPath}

Societe Suisse de Pathologie

Schweizerische Geseluschaft für Pathologie

Swiss Society of Pathology

\section{Schweizerische Gesellschaft für Pathologie}

Schweizerische Gesellschaft für Pathologie

FMH Consulting Services

Burghöhe 1

Postfach 246

CH-6208 Oberkirch

E-Mail:kontakt@sgpath.ch

\section{Jahrestagung der Schweizer Gesellschaft für Pathologie}

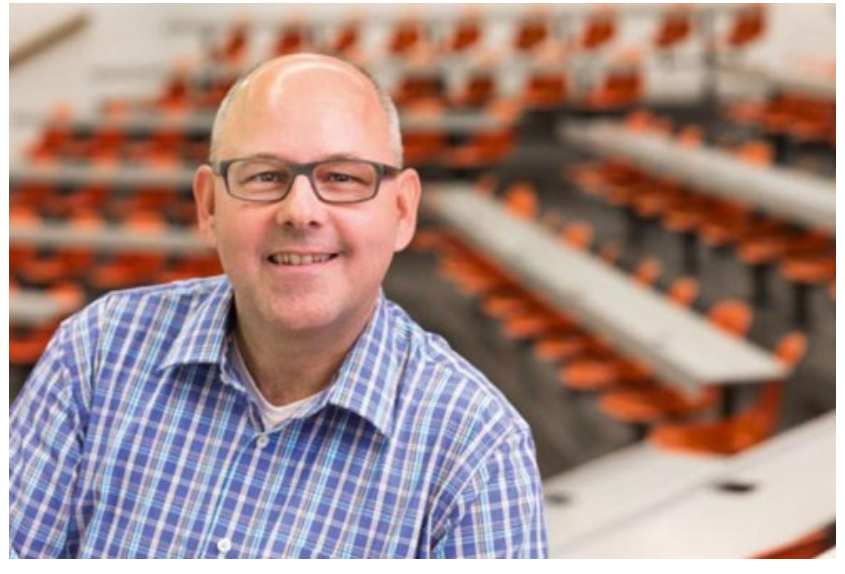

Kongresspräsident Prof. Dr. M. Tolnay

\section{Liebe Kolleginnen und Kol-} legen,

es freut mich, Sie zur 81. Jahrestagung der Schweizer Gesellschaft für Pathologie am 12.-14. November 2015 nach Basel einzuladen.
Die Tagung beginnt mit einem Halbtagsseminar über Dermatopathologie, das sich vor allem an Pathologen in der Ausbildung richtet. Es folgt ein abwechslungsreiches und interessantes wissenschaft- liches Programm, an dessen Gestaltung sich nationale wie internationale Experten beteiligen werden und das aktuelle Themen und Fragestellungen der Pathologie aufgreifen wird. Neben den aktualisierten WHO Klassifikationen der Tumoren der Thoraxorgane sowie des Urogenitaltrakts werden auch Neuigkeiten in der Molekularpathologie sowie der iatrogenen und digitalen Pathologie im Fokus der Jahrestagung stehen. Das wissenschaftliche Programm endet mit einer Keynote Lecture von Prof. Stanley B. Prusiner, der 1997 für die Entdeckung der Prionen mit dem Nobelpreis für Medizin oder Physiologie ausgezeichnet wurde. Ein halbtägiges Schnittse- minar über außergewöhnliche Autopsiebefunde mit interdisziplinärer Diskussion folgt als Abschluss der Jahrestagung.

Alle weiteren Informationen, auch zur Einreichung von Abstracts, folgen in Kürze auf der Homepage der Pathologie am Universitätsspital Basel.

Herzliche Grüße und bis bald in Basel,

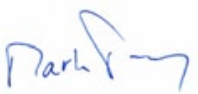

Prof. Dr. med. Markus Tolnay Kongresspräsident 